\title{
Numerical Investigation of Switching Mechanism for the Supersonic Jet Element
}

\author{
Y. $\mathrm{Xu}^{1}$ and G.Q. Zhang ${ }^{1, *}$ \\ ${ }^{1}$ Key Laboratory of Dynamics and Control of Flight Vehicle, School of Aerospace \\ Engineering, Beijing Institute of Technology, Beijing, China, 100081 \\ *Corresponding author: zhanggq@bit.edu.cn
}

\begin{abstract}
ABSTRAC T: Based on the unsteady viscous flow simulation, the flow characteristics inside the supersonic jet element have been investigated numerically. The corresponding initial switching process has been overall divided into two major stages. The results have shown that the switching process for the supersonic jet flow is an extremely complex process, which can include the complex shock system evolution, the free shear layers together with the boundary layers evolution and multiple vortex region unsteady evolution etc. The presence of the switching oblique shock near the control port is not the necessary condition to make the jet deflect, but its formation is good for the early transverse extension of the stripping vortex zone. A new concept named minimum control mass flow rate has also been proposed and emphasized. The Viscosity and adverse pressure gradient have been found to be the key factors for the occurrence of flow separation to shorten the switching time. The vortex structures at different switching time together with the variations (thrust, static pressure as well as oblique shock waves etc.) inside the supersonic jet element have been obtained computationally and analyzed in details.
\end{abstract}

Keywords: Supersonic jet element; shock wave; vortex; shear layer; switching

\section{List of abbreviations}

$$
\begin{aligned}
F & =\text { Total thrust }(N) & e & =\text { Dissipation rate }\left(\mathrm{m}^{2} / \mathrm{s}^{3}\right) \\
k & =\text { Kinetic energy }\left(\mathrm{m}^{2} / \mathrm{s}^{2}\right) & f_{l} & =\text { Left outlet thrust }(N) \\
m & =\text { Mass flow rate }(K) & f_{r} & =\text { Right outlet thrust }(N) \\
M a & =\text { Mach number } & l_{\text {out }} & =\text { Pressure outlet }(P a) \\
P S & =\text { Main gas source pressure }(\text { bar }) & \Delta t & =\text { Time step }(\mathrm{s}) \\
T & =\text { Temperature }(K) & P_{01} & =\text { Point from main jet inlet }(-0.0062,0.042) \\
t & =\text { Switching time }(s) & P_{02} & =\text { Point from main jet inlet }(0.0062,0.042)
\end{aligned}
$$




\section{Introduction}

The supersonic jet element (SJE) is the execution unit in the attitude control system for different spacecrafts. Its performance (such as the control force, switching time, energy efficiency and reliability etc.) can play an extremely important role in controlling the accuracy of the whole space flight[1-3]. The SJE is also called fluidic amplifier. On one hand, it could be used as the logic control system components to achieve a variety of logic functions. On the other hand, through a small energy control signals, it also could be served as an implementation part of the control system and offer much large energy or torque directly[4-7]. Nowadays the supersonic jet element is applied to the Multiple Launch Rocket System project as the implemental components of the control system. Raja[8] had numerically investigated the mixed convection flow and heat transfer characteristics in a two-dimensional plane, laminar offset jet issuing parallel to an isothermal flat plate. The results had shown that the reattachment length is strongly dependent on both the Reynolds number and the Grashof number for the range considered. Sawyer[9] had applied the analysis to the flow due to a jet emerging at an angle to a flat plate and given a good prediction of the length and average pressure of the recirculation region for a particular value of an entrainment-ratio parameter.

Silnikov and Chernyshov[10] had applied the mathematical model of "differential conditions of dynamic compatibility" to theoretically analyze the evolution of the incident shock in the plane overexpanded jet flow or in the axisymmetric one. The corresponding analytical results can be applied to avoid jet flow instability and selfoscillation effects at rocket launch, to improve launch safety and to suppress shock-wave induced noise harmful to environment and personnel. Sary[11] had modeled the plasma synthetic jet actuator's operation. Parametric studies on the geometrical aspects of the actuator as well as its electrical inputs have also been performed, improving its operation and efficiency and resulting in a quasi-periodic pulsed regime. Svensson[12] had used three different turbulence models to predict mean velocity field as well as turbulence characteristics in the near zone of a $6 \times 6$ in-line array of unconfined confluent round jets. The numerical results had been compared with experimental data acquired by Particle Image Velocimetry. The results had shown that the jet's position within the configuration 
has a substantial impact on the velocity decay, length of the potential core, and the lateral displacement of the confluent jets.

However, the switching mechanism of the supersonic jet components still lacks of a deep understanding. The corresponding switching reasons are always simply attributed to the oblique shock and the Coanda effect. Based on this, our present research will further analyze the flow characteristics of the switching process and then reveal the specific switching mechanism for the jet supersonic components.

The paper is organized as follows. Section II describes the governing equations including a brief description of the adopted numerical algorithm. The validation method and results will be also presented and discussed. Section III will specifically discuss the flow field before switching, thrust as well as static pressure distribution during switching, switching mode, formation and evolution of oblique shock, jet leaving the attached wall layer etc. The changes for different parameters in the whole switching process are also investigated in details. The paper will end with brief concluding remarks.

\section{Simulation setup and accuracy test}

\subsection{Simulation setup}

As shown in Fig.1(a), the supersonic jet element (SJE) is a kind of amplifying Element without moving parts which always uses the gas as the working medium. Fig.1(b) shows the work mechanism of the SJE. Compared with the other flexible parts of the amplifier (such as pneumatic or hydraulic slide valve, rotary valve or diaphragm valve), the supersonic jet element has many advantages, such as the low accuracy manufacture and low cost, which also has no moving parts, so the work process does not appear "stuck" in. As implemental components of the control system, the SJE's work performance (control, switching time, energy efficiency, working reliability et al.) has an important role in the control accuracy of the system. Fig.1(c) shows the surface girds of the SJE. In modeling the whole SJE, the quality and size of the mesh generation will directly affect the accuracy of the computational results. So during meshing the structural grids of the SJE, we will adopt the method of size function to control the grid size (It doesn't change the entire structure of the network topology). And the parts of structural 
grid can be refined for the whole Element, and the structural grid can be smoothed and swapped, enhancing the accuracy of the simulation.

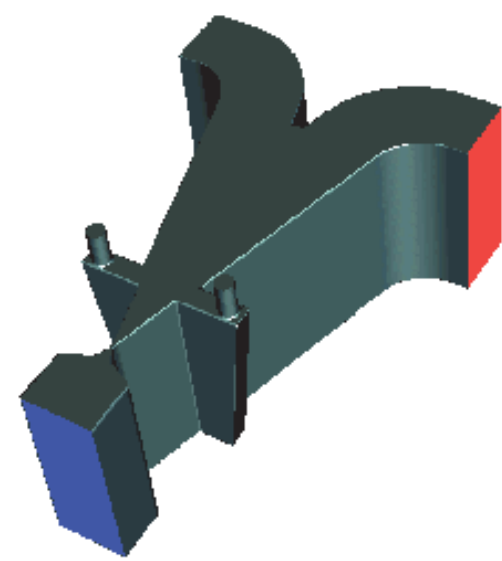

(a) 3D Structure shape

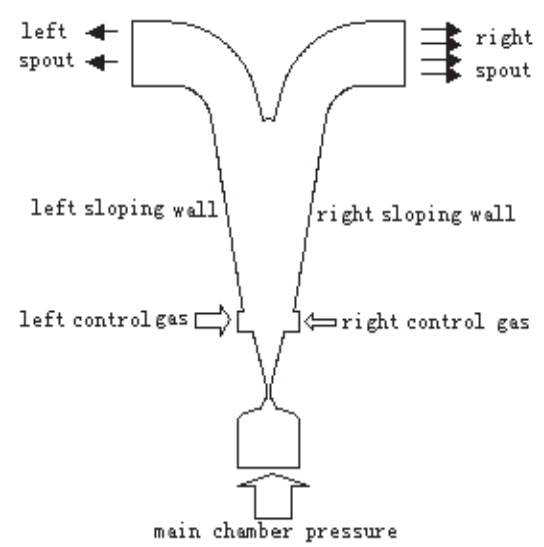

(b) Work mechanism

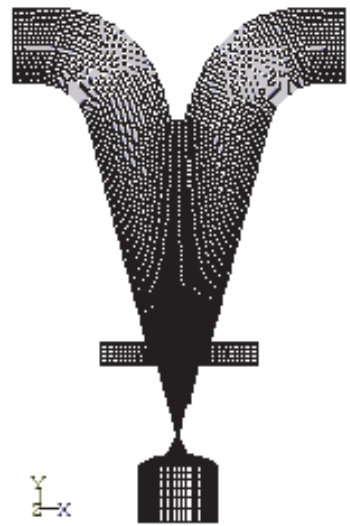

(c) Computational grids(slice)

Fig.1 The work mechanism and grids for the supersonic jet element

The Gauss-Seidel method is used to calculate the inner fluid field of the supersonic jet element. The governing equations of flow are Reynolds Averaging Navier-Stokes equation. The model of turbulence is Realizable k-epsilon model, and Two-layer zonal model is used near the wall.

\subsection{The accuracy test}

The thrust in the attached wall condition is an important measurement to indicate the performance of supersonic jet components good or bad. Therefore, we will first calculate the pressure thrust created by a variety of primary gas source pressure, and then under the same conditions, the thrust value corresponding to the main gas source pressure will be obtained eventually. The verification of the thrust force in the switching process are verified by a single channel air conditioning system[13]. The test system consists of four parts: high pressure and large flow pressure regulating system, data acquisition and processing system, jet element test bench and control table.

As shown in Fig.2, the high pressure and large flow pressure regulating system consists of high pressure compressor (WZ2.3/450) and high pressure gas cylinder group (TGP-50L/35MPa), which will be used to provide the high gas resource and control supersonic airflow. 


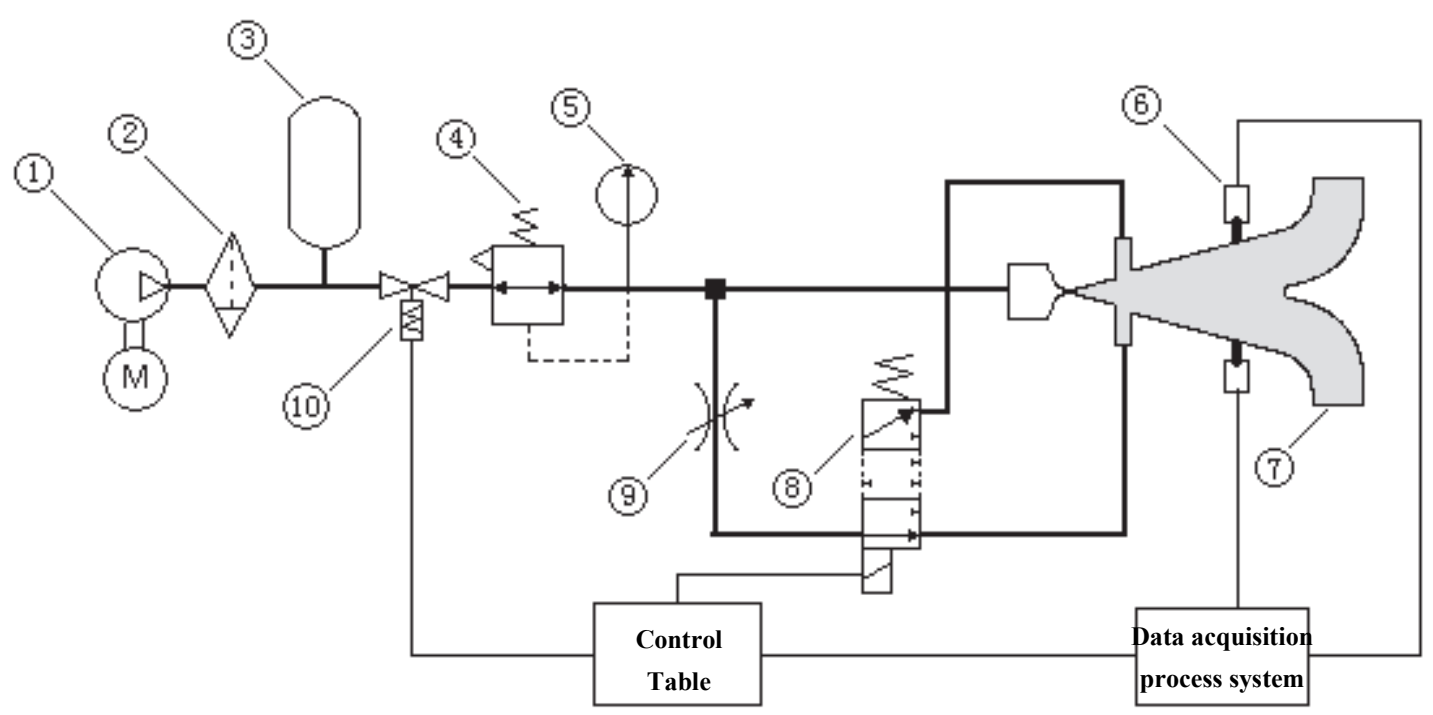

Fig.2 Schematic diagram of the fluidic element test system

where: (1) Gas compress machine, (2)Filter, (3) Gas bottle, (4)Compression release valve, (5) Pressure gauge, (6)Force sensor, (7) SJE, (8) Solenoid directional control valve, (9) Adjustable flow valve, (10) Cut-off valve.

Dynamic test rack is used to fix the fluidic element, and the jet component test bed movable frame is installed with two high frequency and accuracy force sensors. The sensors will be used to monitor the changes of the total thrust during the switching process. The data acquisition and processing system is used to collect the voltage signal from the force sensor and then to process the corresponding noise. The cut-off valve, control valve conversion, data acquisition and processing will be totally controlled by the control table.

Table. 1 The comparison of the calculated and the experimental thrust

\begin{tabular}{|c|c|c|c|c|c|}
\hline \hline $\boldsymbol{P s}(\boldsymbol{b a r})$ & $\boldsymbol{E x p}-\boldsymbol{F}(\boldsymbol{N})$ & $\boldsymbol{f}_{\boldsymbol{r}}(\boldsymbol{N})$ & $\boldsymbol{f}_{\boldsymbol{l}}(\boldsymbol{N})$ & $\boldsymbol{C a l - F ( N )}$ & $\boldsymbol{E r r o r}(\%)$ \\
\hline 20 & 44.0 & 49.65 & 0.42 & 49.23 & 11.89 \\
\hline 30 & 79.9 & 84.34 & 1.95 & 82.40 & 3.13 \\
\hline 40 & 106.0 & 108.91 & 4.43 & 104.48 & -1.43 \\
\hline 45 & 126.3 & 142.09 & 4.58 & 137.51 & 8.87 \\
\hline 50 & 142.6 & 164.59 & 5.66 & 158.93 & 11.45 \\
\hline 55 & 161.4 & 170.27 & 5.46 & 164.81 & 2.11 \\
\hline 60 & 179.3 & 186.87 & 6.43 & 180.44 & 0.64 \\
\hline 70 & 202.8 & 220.68 & 8.55 & 212.12 & 4.6 \\
\hline 80 & 239.9 & 263.05 & 18.04 & 245.01 & 2.1 \\
\hline 90 & 275.4 & 296.36 & 21.78 & 274.58 & -0.3 \\
\hline 100 & 313.2 & 346.18 & 27.89 & 318.29 & 1.63 \\
\hline
\end{tabular}


Table. 1 has showed the thrust corresponding to the main gas source pressure (Ps) and test stands for the total thrust in the $X$ direction of the jet components. $f_{1}$ and $f_{r}$ stand for their own thrusts of two outlets, $\vec{F}=\vec{f}_{l}+\vec{f}_{r}$, Error stands for the relative error between the calculation (F) and test. The maximum and minimum difference can be calculated within $12 \%$ and $0.3 \%$, respectively. Considering the accuracy of the measurement method and the simulation errors, the comparisons are showing reasonable overall quantitative agreement, showing the accuracy of the current simulation method.

\subsection{Grid Sensitivity Test}

The accumulation of numerical error and dependence of simulation results on grid size should be studied for the particular problem. For each particular problem accumulation of errors could be different. Besides, for one and the same simulation it is different for different stages of simulations being the function of the number of time steps. Therefore, estimating precision and errors accumulation are extremely necessary for large simulations of the complex fluid problem. And accumulation of error is proportional to the square rote of the number of time steps. It should be evaluated for each numerical simulation, especially for the unsteady flow state, which has been investigated by Smirnov[14-15] in details. The corresponding research had revealed that the relative error

of integration in $1 \mathrm{D}$ case can be simplified as: $S_{1} \approx\left(1 / N_{1}\right)^{k+1}$ in the uniform grid, where $\mathrm{N}_{1}$ is the number of cells in the direction of integration and $\mathrm{k}$ is the order of accuracy of numerical scheme. Then it can be summed up as:

$$
S_{\text {err }} \approx \sum_{i=1}^{3} S_{i}
$$

The allowable value of total error $S^{\max }$ can be presumed as $5 \%$, then the maximum allowable number of time steps can be determined as:

$$
n_{\max }=\left(S^{\max } / S_{e r r}\right)^{2}
$$

where $\mathrm{n}$ is the number of time steps in Navier-Stokes equations integration. The ratio of maximal allowable number of time steps is introduced, which can be used to characterize the reliability of results. the higher the value of $n_{\max }$ is, the lower the error is .

$$
R_{s}=n_{\max } / n
$$


The detailed analysis for the accumulation of error on different combinations (iteration time step and grid resolution) has been shown in Table.2. The accumulation of error for different grid resolution and physical time simulations has been discussed in details. The accumulation of error will begin in a fast manner for coarse grid and decreases on the increasing grid resolution and scheme accuracy, but decrease when the physical time increases.

Table.2 Error estimate

\begin{tabular}{|c|c|c|c|c|c|c|}
\hline $\begin{array}{c}\text { Allowable error } \\
(\%)\end{array}$ & $\begin{array}{l}\text { Grid resolution } \\
(\mathrm{X} \times \mathrm{Y} \times \mathrm{Z})\end{array}$ & $\begin{array}{l}\text { Physical time } \\
\text { simulated (s) }\end{array}$ & $\begin{array}{l}\text { Number of } \\
\text { time steps }\end{array}$ & $\begin{array}{c}\text { Accumulated } \\
\text { error }\end{array}$ & $\begin{array}{c}\text { Allowable number of } \\
\text { time steps }\end{array}$ & $\begin{array}{l}\text { Reliability } \\
R_{S}=n_{\max } / n\end{array}$ \\
\hline 5 & $260 \times 80 \times 80$ & 0.1 & 5000 & 0.00028284 & $1.59 \cdot 10^{8}$ & $3.18 \cdot 10^{4}$ \\
\hline 5 & $500 \times 160 \times 160$ & 0.1 & 10000 & 0.00005000 & $1.02 \cdot 10^{10}$ & $1.02 \cdot 10^{6}$ \\
\hline 5 & $1000 \times 320 \times 320$ & 0.1 & 18182 & 0.00000836 & $6.50 \cdot 10^{11}$ & $3.57 \cdot 10^{7}$ \\
\hline 5 & $1500 \times 640 \times 640$ & 0.1 & 35714 & 0.00000151 & $3.98 \cdot 10^{13}$ & $1.11 \cdot 10^{9}$ \\
\hline 5 & $260 \times 80 \times 80$ & 1.0 & 55556 & 0.00094280 & $1.59 \cdot 10^{8}$ & $2.86 \cdot 10^{3}$ \\
\hline 5 & $500 \times 160 \times 160$ & 1.0 & 90909 & 0.00015076 & $1.02 \cdot 10^{10}$ & $1.12 \cdot 10^{5}$ \\
\hline 5 & $1000 \times 320 \times 320$ & 1.0 & 178572 & 0.00002620 & $6.50 \cdot 10^{11}$ & $3.64 \cdot 10^{6}$ \\
\hline 5 & $1500 \times 640 \times 640$ & 1.0 & 384616 & 0.00000496 & $3.98 \cdot 10^{13}$ & $1.03 \cdot 10^{8}$ \\
\hline
\end{tabular}

As shown in Table.2, for present simulations all the results demonstrate high reliability of the used program. but the number of time steps and physical time should not be chosen too large in order to avoiding the accumulation of error in the whole simulation process. Therefore, keeping the certain iteration accuracy and simultaneously avoiding too long simulated period should be the top priority. After the carefully consideration, we will adopt the relatively moderate computation domain size: $1000 \times 320 \times 320$ in the subsequent computations.

\subsection{Setup for the inlet control flow}

Table. 3 has shown the specific setup for the parameters of the control flow. Due to the fact that the switching time for the supersonic jet element operation is extremely short, the corresponding control signals will always show the typical quati-step functions, so the mass flow rate and static pressure for the both left and right inlet control flows can be assumed as a ramp function within a very short period $(\Delta t)$ at the beginning of the switch, which can be written as:

$$
m_{l}=\left\{\begin{array}{ccc}
m_{1} & t<0^{-} \\
m_{1}+\frac{t}{\Delta t}\left(m_{2}-m_{1}\right) & 0 \leq t \leq \Delta t \\
m_{2} & t>\Delta t
\end{array} \quad m_{r}=\left\{\begin{array}{cc}
m_{2} & t<0^{-} \\
m_{2}+\frac{t}{\Delta t}\left(m_{1}-m_{2}\right) & 0 \leq t \leq \Delta t \\
m_{1} & t>\Delta t
\end{array}\right.\right.
$$




$$
p_{l}=\left\{\begin{array}{cc}
p_{1} & t<0^{-} \\
p_{1}+\frac{t}{\Delta t}\left(p_{2}-p_{1}\right) & 0 \leq t \leq \Delta t \\
p_{2} & t>\Delta t
\end{array} \quad p_{r}=\left\{\begin{array}{cc}
p_{2} & t<0^{-} \\
p_{2}+\frac{t}{\Delta t}\left(p_{1}-p_{2}\right) & 0 \leq t \leq \Delta t \\
p_{1} & t>\Delta t
\end{array}\right.\right.
$$

where $m_{l}$ and $m_{r}$ stands for the mass flow rate on left and right inlet control flow respectively, $p_{l}$ and $p_{r}$ will stand for the corresponding static pressure. $m_{l}=0.08 \mathrm{~kg} / \mathrm{s}$, $m_{2}=0.003 \mathrm{~kg} / \mathrm{s}, p_{1}=210000 \mathrm{~Pa}, p_{2}=57000 \mathrm{~Pa} . \Delta t$ is decided by the ratio of $p_{1}$ and $p_{2}$, here $\Delta t=1 \times 10^{-5} \sim 2 \times 10^{-5} \mathrm{~s}$.

Table.3 Setup for the parameters of the control flow

\begin{tabular}{|c|c|c|c|c|c|c|}
\hline $\begin{array}{c}\text { Cas } \\
\mathrm{e}\end{array}$ & $\begin{array}{c}\text { Boundary } \\
\text { type }\end{array}$ & $\begin{array}{l}\text { Total pressure or } \\
\text { mass flow rate }\end{array}$ & $\begin{array}{c}\text { Static } \\
\text { pressure }\left(10^{5} \mathrm{~Pa}\right) \\
\end{array}$ & $\begin{array}{c}\text { Temperature } \\
(\mathrm{K})\end{array}$ & $\begin{array}{l}\text { Turbulence } \\
\text { parameters }\end{array}$ & Remarks \\
\hline Ps & $\begin{array}{l}\text { Pressure } \\
\text { inlet }\end{array}$ & $\begin{array}{c}100 \times 10^{5} \\
(\mathrm{~Pa})\end{array}$ & 99.8 & 300 & $\begin{array}{c}\text { Turbulence } \\
\text { intensity: } 4 \% \\
\text { Hydraulic } \\
\text { diameter: } \\
0.0384(\mathrm{~m})\end{array}$ & - \\
\hline $\mathrm{m}_{1}$ & \multirow{2}{*}{$\begin{array}{l}\text { Mass-flow } \\
\text { inlet }\end{array}$} & $0.08(\mathrm{~kg} / \mathrm{s})$ & 2.1 & \multirow{2}{*}{300} & \multirow{2}{*}{$\begin{array}{c}\text { Turbulence } \\
\text { intensity: } 6 \% \\
\text { Hydraulic } \\
\text { diameter: } \\
0.012(\mathrm{~m})\end{array}$} & Positive $\mathrm{X}$-axial \\
\hline $\mathrm{m}_{\mathrm{r}}$ & & $0.003(\mathrm{~kg} / \mathrm{s})$ & 0.57 & & & Negative $\mathrm{X}$-axial \\
\hline $1_{\text {out }}$ & $\begin{array}{c}\text { Pressure } \\
\text { outlet }\end{array}$ & - & 1.0144 & 300 & $\begin{array}{l}k=1\left(\mathrm{~m}^{2} / \mathrm{s}^{2}\right) \\
e=1\left(\mathrm{~m}^{2} / \mathrm{s}^{3}\right)\end{array}$ & reflux conditions \\
\hline
\end{tabular}

\section{Results and discussion}

\subsection{Flow field before switching}

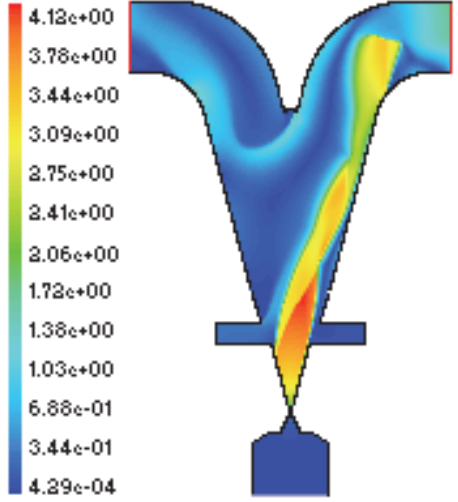

(a) Mach number (Ma)
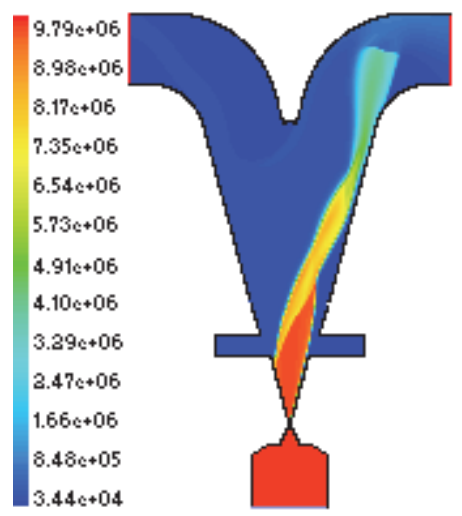

(b) Total pressure $(\mathrm{Pa})$

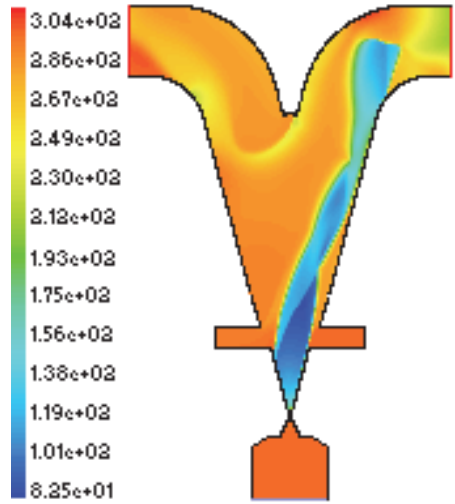

(c) Temperature (K)

Fig.3 The distributions for the jet Element before switching 
Before switching, the left control port mass flow rate is greater than that of the right, the main jet will flow along the wall layers, and most of the jet flow will be ejected from the right outlet. As shown in Fig.3(a), the velocity distributions on the right outlet are always larger than the left outlet. The corresponding total pressure distributions are also larger than left ones(shown in Fig.3(b). So the corresponding static temperature regions are lower than the left outlet(shown in Fig.3(c)).

\subsection{The changes on the thrust static reference point during the switching process}

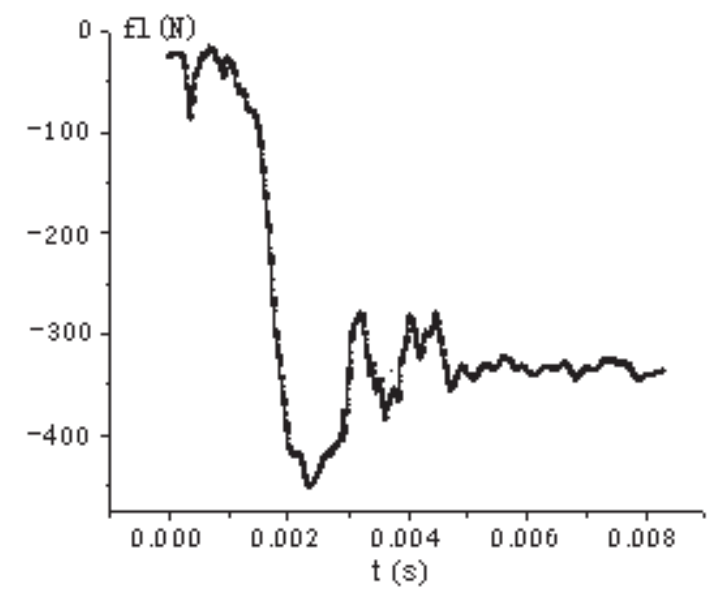

(a) Left outlet

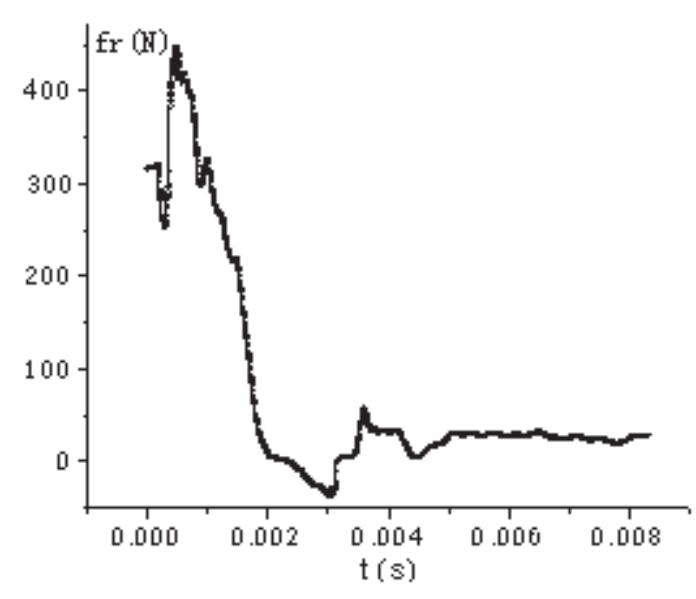

(b) Right outlet

Fig.4 The thrust for the left and right outlets

As shown in Figs.4(a) and (b), due to the effects from the fluid viscous diffusion processes in the supersonic jet Element, the lagged effect of acoustic wave propagation and whirlpool in compressible flow processes, free shear layer, boundary layer evolution etc., the left and right output flow parameters can not immediately respond to changes of the input. And shortly after the change of the control flow inlet conditions, the thrust for the right outlet will be reduced, while the left outlet will be increased, which can generate a brief diversion phenomenon. Except this, before the main jet begins to deflect, the right output port has generated an overshoot thrust. When the main jet completes the deflect motion, the left output port will also generate the overshoot thrust. This can make the great impact on the attitude control engines, rocket-bomb body and then directly affect the control accuracy and system reliability.

During the deflection, the left output thrust has a bigger linear range comparing with the right. And the thrust for the left output will always show the increasing trend, while 
the right is decreasing gradually. After finishing the deflection motion, both the thrust for the left and right outputs still need a certain time to recovery in order to form the steady flow along the wall layers.

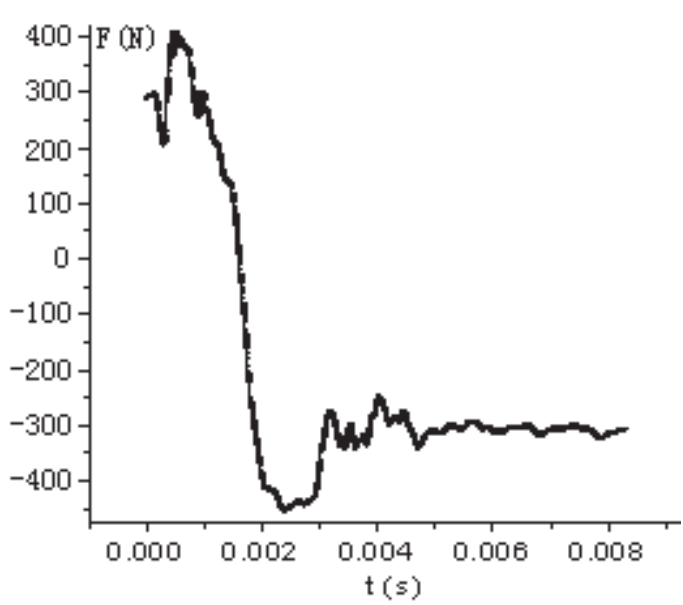

(a) Changes during the switching process

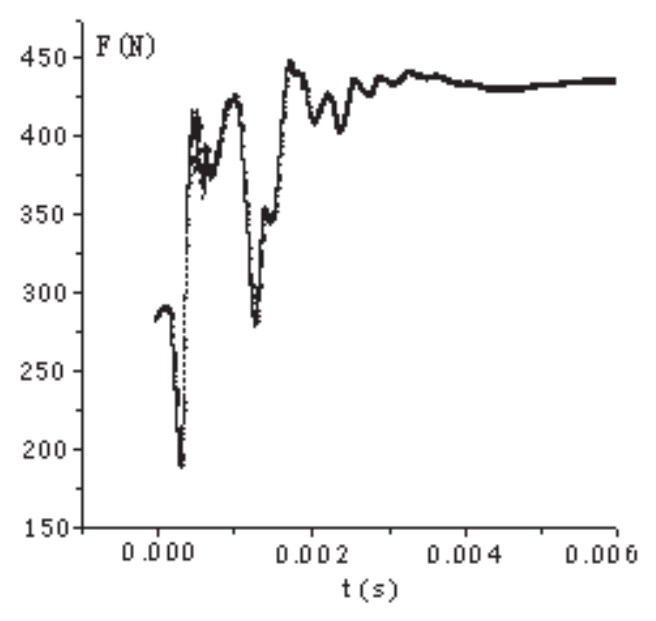

(b) Changes in the insufficient mass flow rate

Fig.5 The changes for the combined thrust

As shown in Fig.5, the positive portion for the combined thrust curve is similar to the upper curve of the right thrust, while the negative portion is similar to the lower curve of the left thrust. It has indicated that the size of the combined thrust is mainly decided by the right output thrust before the main jet flow begins to deflect. After the deflection, the combined thrust will be totally decided by the left output thrust. As shown in Fig.5(a), defining the combined thrust before switching as the positive value, the positive overshoot thrust before the switching is about $30 \%$ bigger compared with the steady state. After finishing the switching process, the negative overshoot thrust has become about 50\% bigger compared with the steady state. For a given jet components, there will exist a minimum control flow mass flow rate. If the control flow of the mass flow rate is less than the minimum value, the corresponding switching process will not be completed successfully and the direction of the combined thrust will also remain unchanged. As shown in Fig.5(b), the switching control command will be changed at $\mathrm{t}=0 \mathrm{~s}$, however, due to the insufficient mass flow rate, the jet flow will not be switched according the controlling command, the generated combined thrust always remains positive. In this case, the combined thrust after the oscillation period of adjustment will tend to be steady, which is greater than the combined steady thrust value before switching. 
In order to observe the static pressure changes on the left and right inlet control flows, both the $\mathrm{P}_{01}(-0.0062,0.042)$ and $\mathrm{P}_{02}(0.0062,0.042)$ have been picked from the lower edge of the main jet inlet. Figs.6(a) and (b) has respectively shown the static pressure changes on the two chosen points.

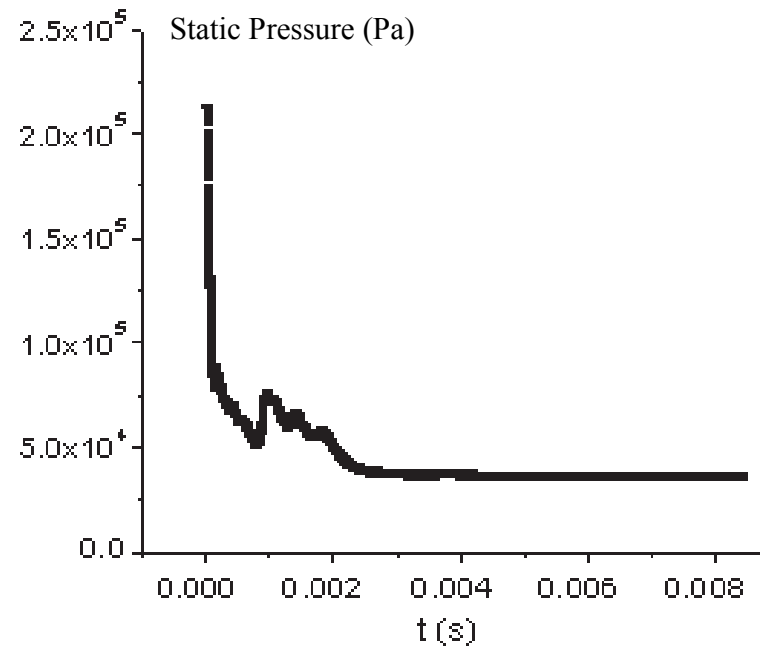

(a) $\mathrm{P}_{01}$ case

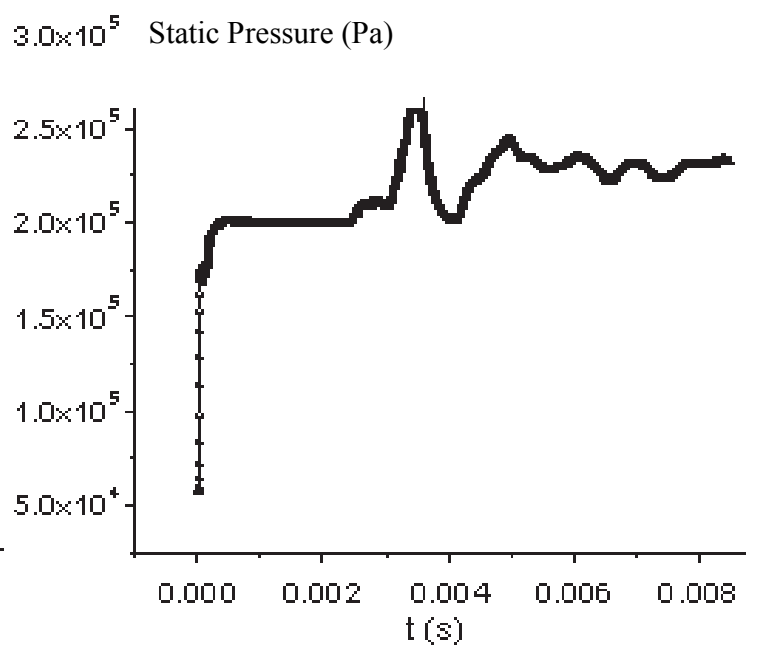

(b) $\mathrm{P}_{02}$ case

Fig. 6 The changes of the static pressure

As shown in Fig.6, in a very short time after the inlet control flow changed, the static pressure for $\mathrm{P}_{01}$ has experienced a sharp decrease, while the $\mathrm{P}_{02}$ is totally reversed. After a certain oscillation period $(\mathrm{t}>3.6 \mathrm{~ms})$, the static pressure at $\mathrm{P}_{01}$ tends to be a small hydrostatic (about $3.6 \times 10^{5} \mathrm{~Pa}$ ). However, to be different from $\mathrm{P}_{01}$, the static pressure at $\mathrm{P}_{02}$ has increased by $2 \times 10^{5} \mathrm{~Pa}$, and then become steady for a long time. When $\mathrm{t}>2.5 \mathrm{~ms}$, the corresponding value will begin to oscillate. The maximum oscillating amplitude can be ranged from 3 to $5.5 \mathrm{~ms}$, the maximum has reached about $2.74 \times 10^{5} \mathrm{~Pa}$. When $\mathrm{t}>5.5 \mathrm{~ms}$, the static pressure will oscillate near $2.28 \times 10^{5} \mathrm{~Pa}$.

\subsection{Switching mechanism}

In the present study, the switching time will be defined as the period from the control flow of the elements begin to change to the supersonic jet can generate the relatively stable reverse thrust. This process will experience an extremely complex process. For better to investigate this complex unsteady flow process, our present studies will be divided into two stages, i.e., i) The switching oblique shock wave formation and 
boundary layer separation (Stage I); ii) The jet starts to separate from the right side of the wall layer (Stage II). The specific analysis will be given as follows.

\subsubsection{The switching oblique shock wave formation and boundary layer separation} stage(Stage I)

The initial time for this stage will be defined as the moment that the control signal starts to change (i.e., the left control flow begins to decrease while the right control flow starts to increase). The terminal time can be defined as the moment that the attached wall starts to move forward to the positive $\mathrm{Y}$ direction.

(a) The establishment of the pressure

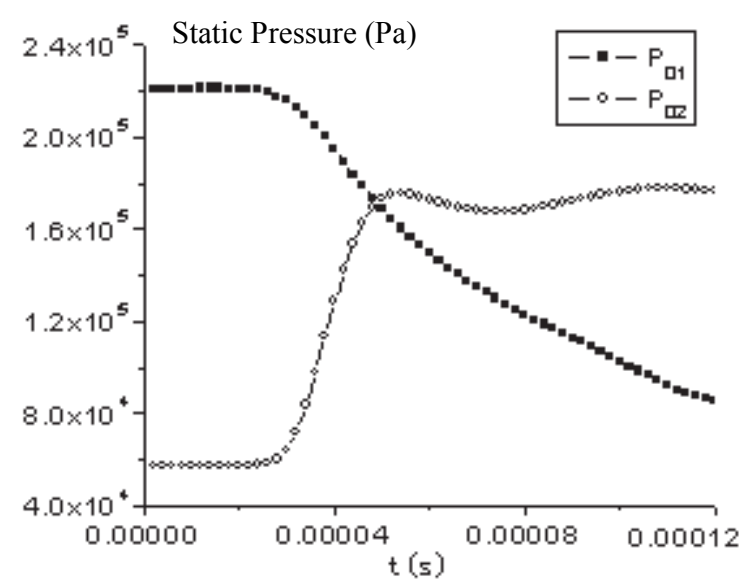

(a) The pressure changes at $\mathrm{P}_{01}$ and $\mathrm{P}_{02}$

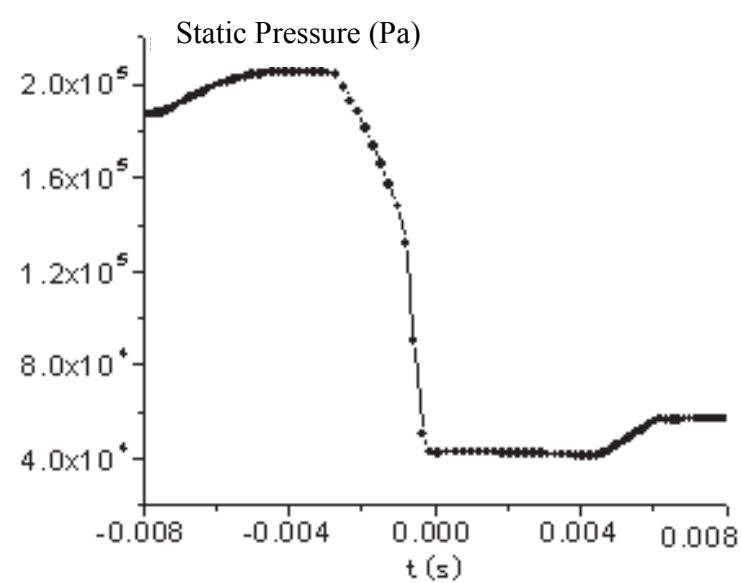

(b) The static pressure distribution at $\mathrm{Y}=0.047 \mathrm{~m}$

Fig.7 The static pressure changes for the studied points and iso-surface

As shown in Fig.7(a), after the controlling signal changed and about $2.5 \times 10^{-5} \mathrm{~s}$ delaying, the left entrance $\left(\mathrm{P}_{01}\right)$ of the pressure control flow has been gradually reduced while the right one $\left(\mathrm{P}_{02}\right)$ has been increased sharply. When $\mathrm{t}>5 \times 10^{-5} \mathrm{~s}, \mathrm{P}_{01}$ will continue decreasing. However, the increasing trend for $\mathrm{P}_{02}$ has been stopped and the corresponding value will fluctuate around $1.8 \times 10^{5} \mathrm{~Pa}$. In this case, the control flow coming from the right inlet will be mainly used to expand the stripping vortex area. In addition, for the selected iso-surface $(\mathrm{Y}=0.047 \mathrm{~m}$ ) shown in Fig.7(b), the corresponding static pressure has experienced a sharp decrease around $\mathrm{t}=0 \mathrm{~s}$, then the curve will experience gradually increasing trend after $\mathrm{t}>0.004 \mathrm{~s}$. 


\section{(b) The formation and evolution of oblique shock at switching control flow}

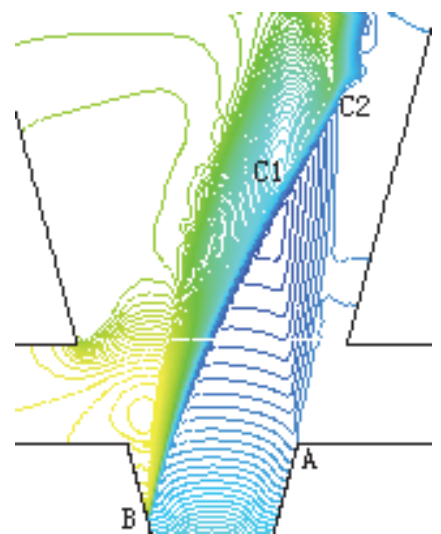

(a) Before switching

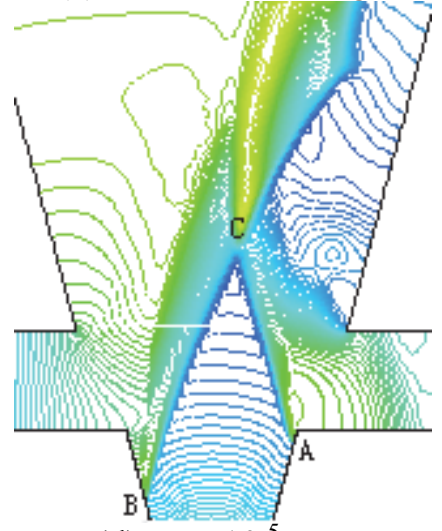

(d) $\mathrm{t}=7 \times 10^{-5} \mathrm{~s}$

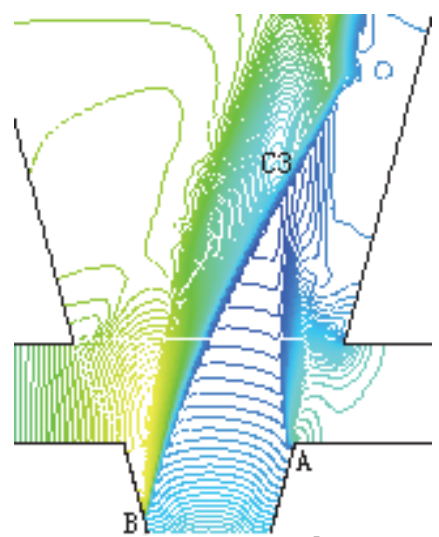

(b) $\mathrm{t}=4 \times 10^{-5} \mathrm{~s}$

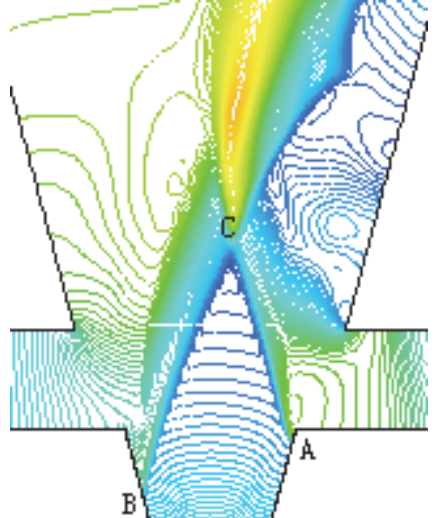

(e) $\mathrm{t}=8 \times 10^{-5} \mathrm{~s}$

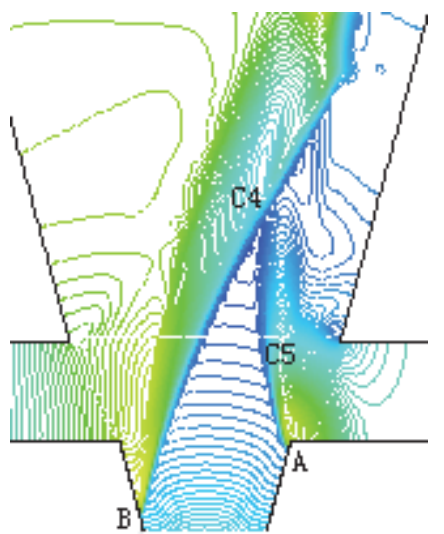

(c) $\mathrm{t}=5 \times 10^{-5} \mathrm{~s}$

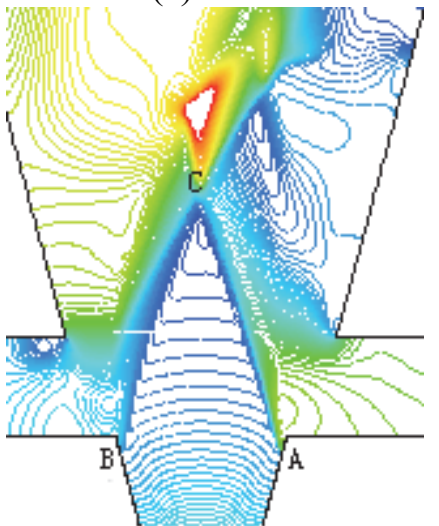

(f) $\mathrm{t}=1.2 \times 10^{-4} \mathrm{~s}$

Fig. 8 The formation and evolution of switching oblique shock

As shown in Fig.8(a), before switching, there exists a wide and weak compression wave near the left control inlet $\left(\mathrm{AC}_{1}-\mathrm{AC}_{2}\right)$. With the right control flow involving into the stream, the pressure at the right entrance will increase, the strength of the compression wave will also increase, and the original wide weak compression wave has become a narrower but stronger compression wave $\left(\mathrm{AC}_{3}\right)$ shown in Fig.8(b). With the pressure continues increasing, the strength of the compression wave at point A will further increase and then form a shock wave $\left(\mathrm{AC}_{5}\right)$. At $\mathrm{t}=7 \times 10^{-5} \mathrm{~s}$, the compressed wave (C5C4) will be transferred to an oblique shock wave (AC) shown in Fig.8(d).

In addition, as shown in Figs.8(d), (e) and (f), due to the pressure is not very large at the left entrance in the later stage I, so both the angle of the switching oblique shock wave and position changes of point A are extremely little. However, with the oblique shock wave at left entrance continuously weakening, the switching obligue shock wave (AC) will move gradually to the upper left direction. 


\section{(c) Boundary layer separation}

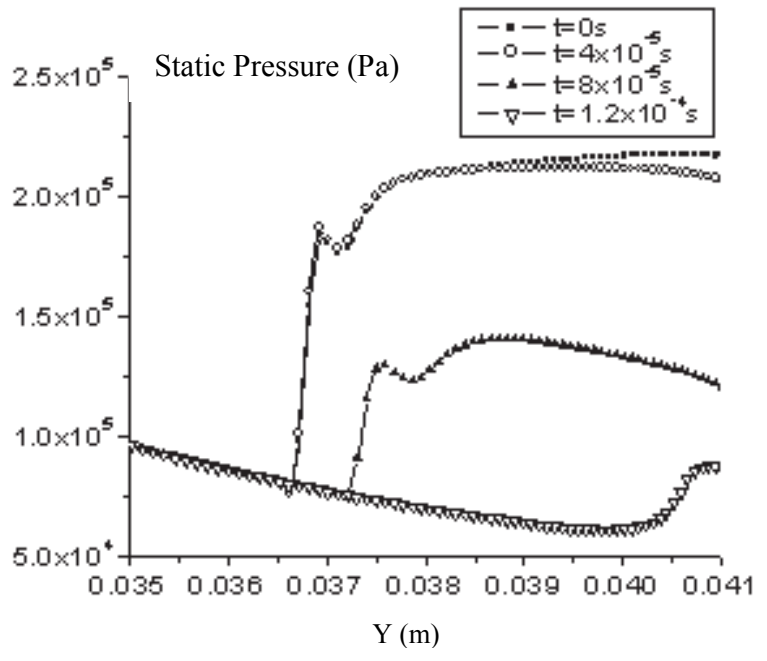

(a) The static pressure distributions on the wall $\left(1_{1}\right)$

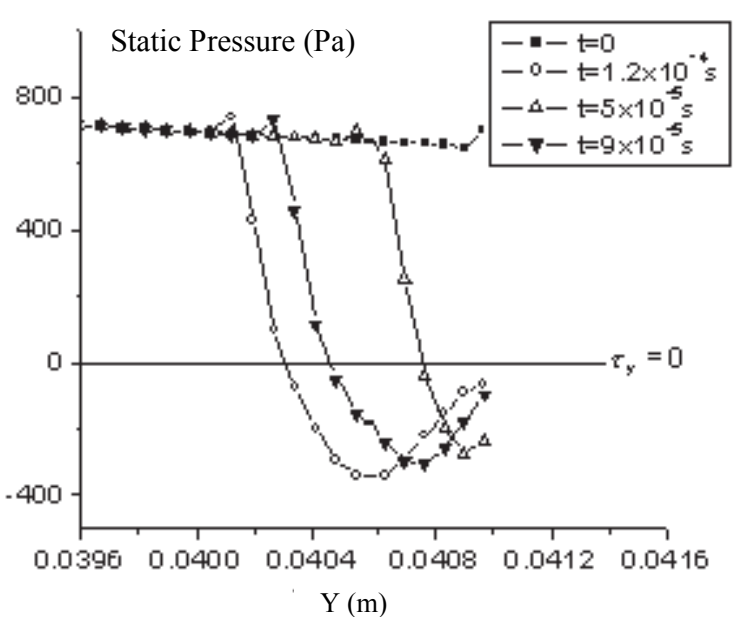

(b) Shear stress distribution on the wall $\left(\mathrm{r}_{1}\right)$

Fig.9 The static pressure and shear stress distributions on the wall layers

Fig.9(a) has shown the static pressure distributions on the wall $\left(1_{1}\right)$ at different time. At $\mathrm{t}=0 \mathrm{~s}$ and $\mathrm{t}=4 \times 10^{-5} \mathrm{~s}$, the position and strength of the shock wave are similar. With the time increasing, the pressure at left entrance will decrease gradually, which can make the pressure difference for the oblique shock wave at wall $1_{1}$ become weaker. The strength and angle of shock BC will also be reduced. When $t=4 \times 10^{-5}$ s, the shock $\mathrm{BC}$ has been changed to a weaker compressed wave. Except this, the corresponding position has also been changed (Point B is moving to the lower edge of the left entrance).

The flow separation will always cause the loss of the energy, larger drag and the lower efficiency. Therefore, a lot of work will always focus on how to avoid the flow separation phenomena. An important factor to measure the performance is good or bad is the length of the switching time. In order to shorten the switching time, the control flow should be separated as soon as possible so as to make the main jet deflect to the opposite side in a short time. Viscosity and adverse pressure gradient are the necessary condition for the occurrence of flow separation. After the switching shock wave formed, the stronger adverse pressure gradient will be created at the downstream, which can cause the flow separation. For the separation point, the following relationship will exist:

$$
\left(\frac{\partial u}{\partial y}\right)_{y=0}=0
$$


As shown in Fig.9(b), before the controlling signal starts to change, the shear stress distribution on the wall $\left(\mathrm{r}_{1}\right)$ in Y-direction will always keep positive. However, once the signal changes, the corresponding value will quickly jump to negative. Fig.9(b) has also shown the separation point $\left(\tau_{\mathrm{y}}=0\right)$. With the strength of the oblique shock wave increasing, the separation point will start to leave the right entrance.

\section{(d) The changes of the stripped vortex zone}

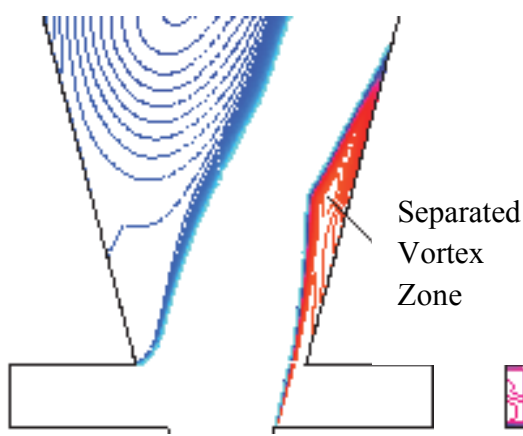

(a) Before switching

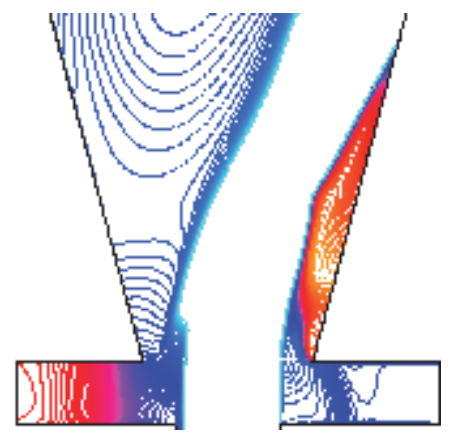

(d) $\mathrm{t}=7 \times 10^{-5} \mathrm{~s}$

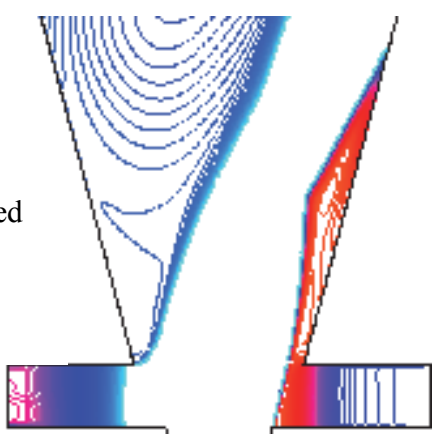

(b) $\mathrm{t}=3 \times 10^{-5} \mathrm{~s}$

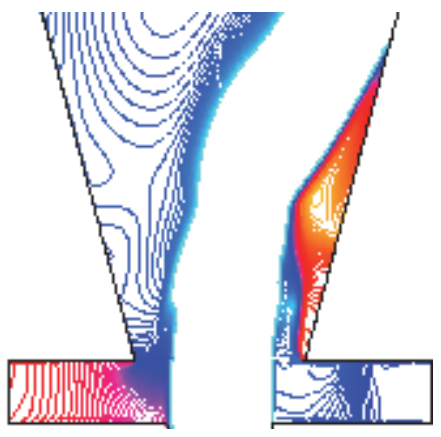

(e) $\mathrm{t}=9 \times 10^{-5} \mathrm{~s}$

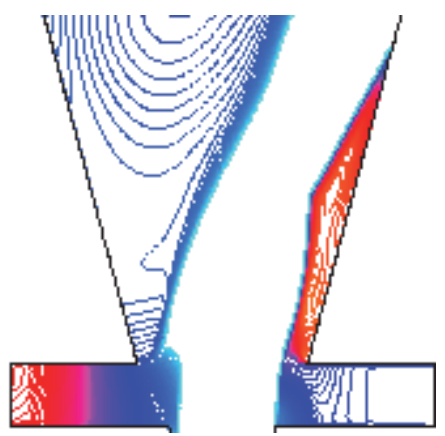

(c) $\mathrm{t}=5 \times 10^{-5} \mathrm{~s}$
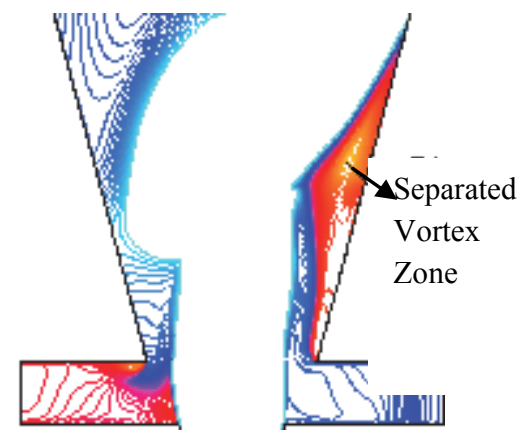

(f) $\mathrm{t}=1.2 \times 10^{-4} \mathrm{~s}$

Fig. 10 The stripped vortex zones at different switching time

Once the controlling signal starts to change, the pressure at right entrance will be increased and form a switching oblique shock wave. As shown in Figs.10(a) (c), before $\mathrm{t}=5 \times 10^{-5}$, the size, shape and total pressure changes at the vortex core are extremely small. When $t>5 \times 10^{-5}$, the stripped vortex zone will be expanded in X-direction. And the corresponding shape and size have also been changed(shown in Figs.10(d) (f)). The total pressure at vortex core has also reduced while the position of the attaching point still has no changes. 
(e) Main jet flow into the main vortex area

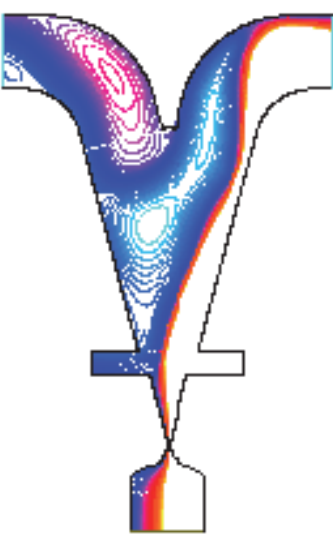

(a) Before switching

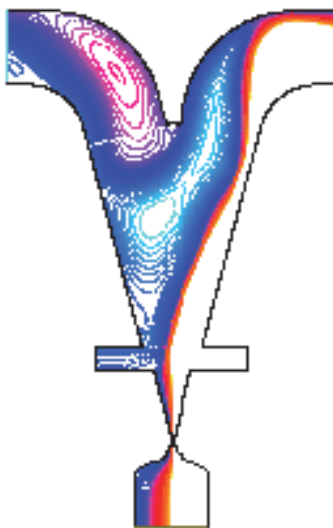

(b) $t=4 \times 10^{-5} s$

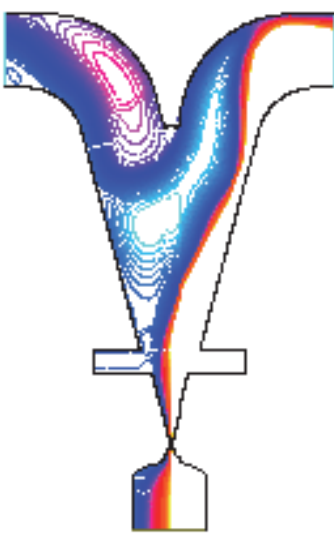

(c) $\mathrm{t}=8 \times 10^{-5} \mathrm{~s}$

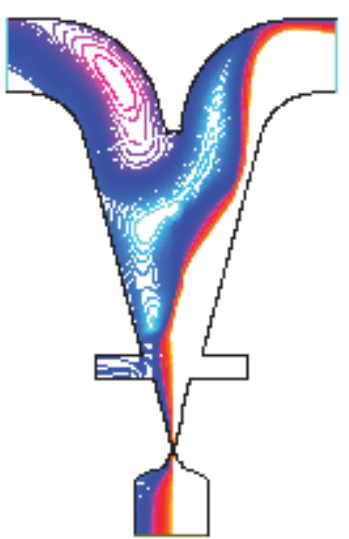

(d) $\mathrm{t}=1.2 \times 10^{-4} \mathrm{~s}$

Fig.11 The position changes of the main vortex area

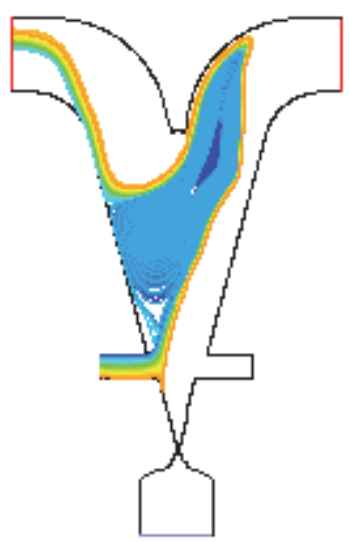

(a) Before switching

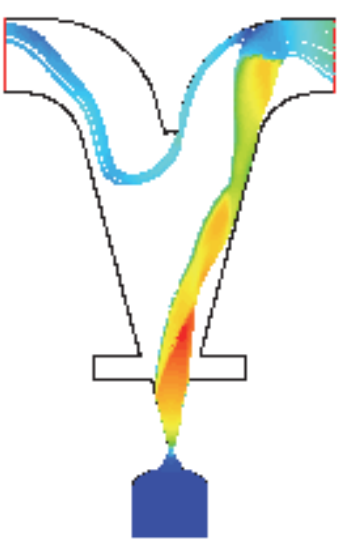

(b) $\mathrm{t}=4 \times 10^{-5} \mathrm{~s}$

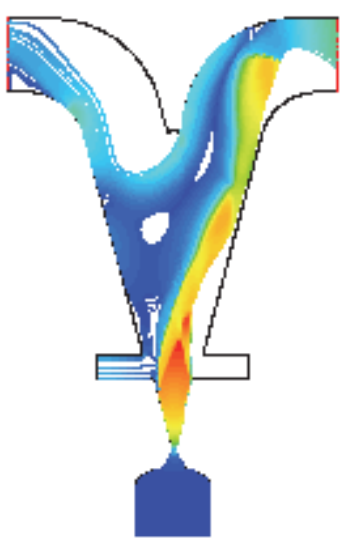

(c) $\mathrm{t}=8 \times 10^{-5} \mathrm{~s}$

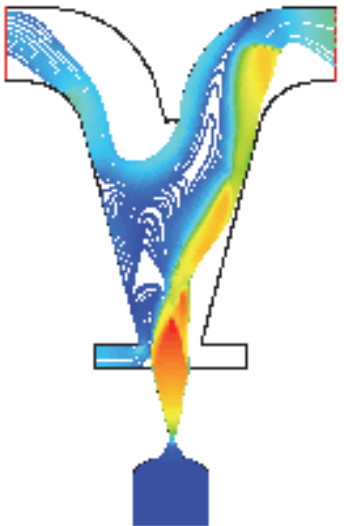

(d) $\mathrm{t}=1.2 \times 10^{-4} \mathrm{~s}$

Fig.12 Distribution of the left control flow and main jet transient spatial

Fig.11 has shown the changes of the primary vortex zone in phase I. Before switching, the airflow in the primary vortex zone has been fiercely swept away. At mean time, part of the left controlling air has also flown into the primary vortex zone (As shown in Fig.11(a) and Fig.12(a)). When the jet Element is under the attaching state, the mass for the swept air will become equal to the newly involved controlling air. The pressure of the primary vortex region will be kept in balance. As shown in Fig.11(c) and Fig.12(c), when the controlling signal has changed, the air mass flow rate for the left control port input will decrease rapidly, while the air in the primary vortex zone will continue to be swept away. Due to lacking of a sufficient number of control flow to replenish the swept air, the 
pressure in the primary vortex zone will be decreased and the original pressure equilibrium will also be destroyed. Affected by the pressure difference, part of the primary jet will flow into the primary vortex zone.

\subsubsection{Jet leaving the right attached wall layer (Stage II)}

The initial time for stage II will be defined as the moment that the attached point starts to move to the positive $\mathrm{Y}$ direction. The ending time will be defined as the moment that the right side of the jet starts to leave the right attached wall layer (including the right wall and attached convex wall).

\section{(a) The position changes of the attached point}

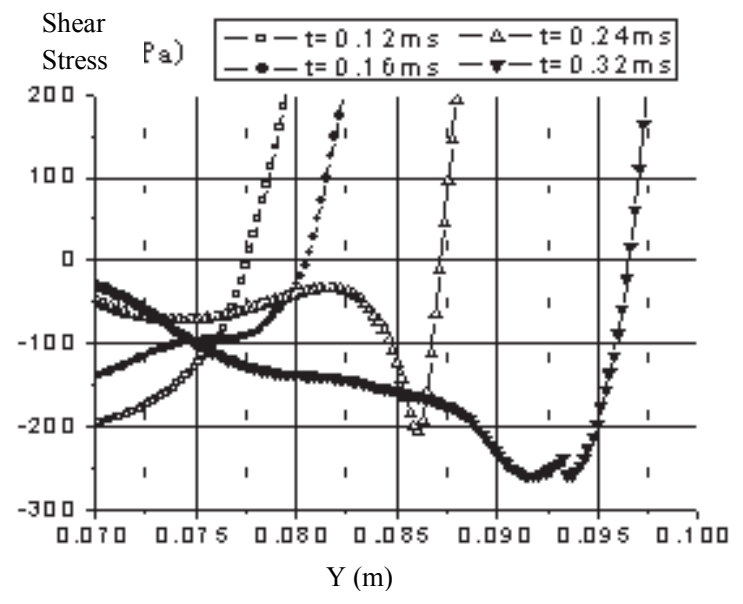

(a) $\mathrm{t}=0.12 \sim 0.32 \mathrm{~ms}$

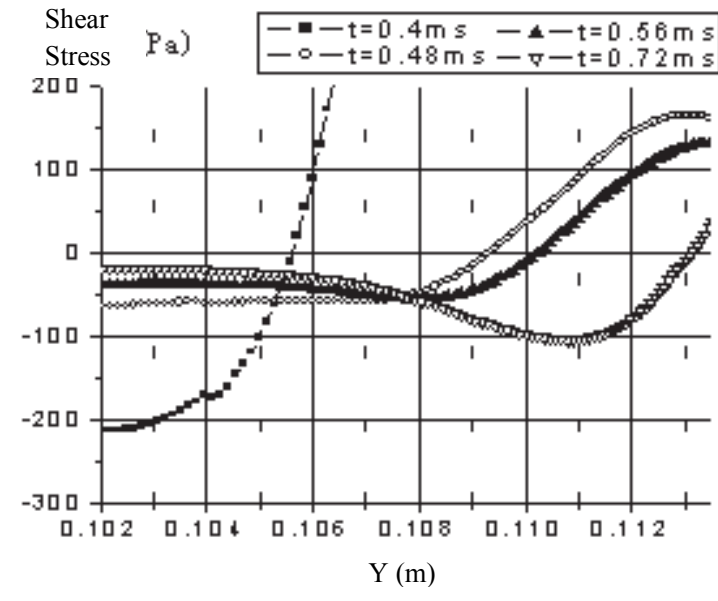

(b) $\mathrm{t}=0.4 \sim 0.72 \mathrm{~ms}$

Fig. 13 The position changes of the attached point at different time $(\mathrm{t}=0.12 \sim 0.72 \mathrm{~ms})$

As shown in Fig.13, due to the fact that attached point is the demarcation point for downstream flows and reverse separated vortex region, so the shear stress at this point should be zero in the Y direction. As shown in Figs.13(a) and (b), with the time increasing, the attached point has moved gradually to the position Y direction in stage II, which will indicate that the position of the attached point is moving to the downstream with the time increasing. 


\section{(b) The changes for the left and right thrust}

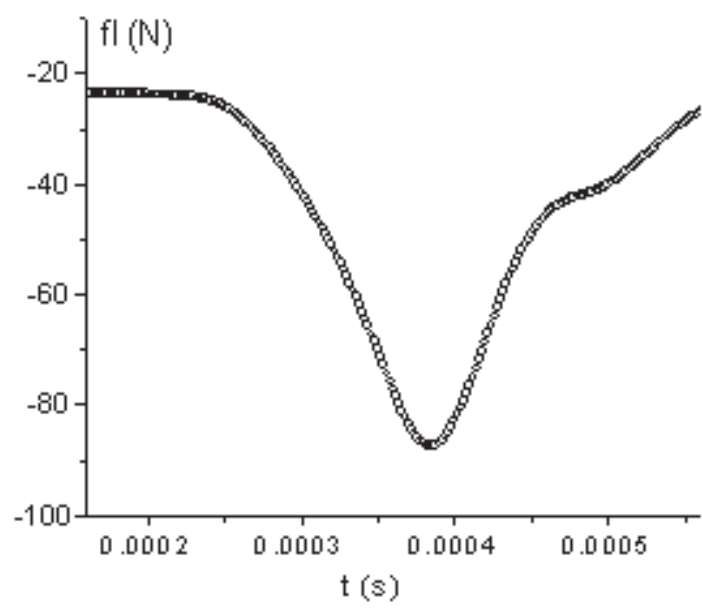

(a) Left thrust

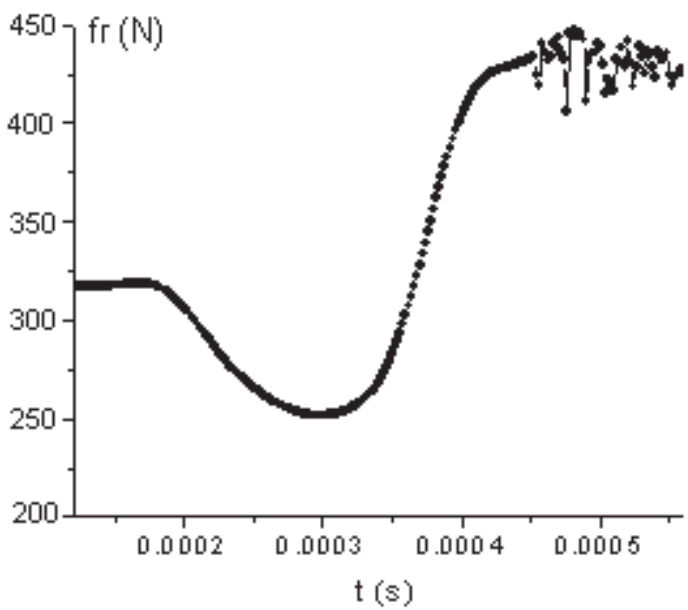

(b) Right thrust

Fig.14 The changes for the left and right thrust in stage II

As shown in Figs.14(a) and (b), after the short time of the controlling signal begins to change, the thrust for the left output has increased while the right case has decreased. This phenomena is mainly due to the fact that part of the primary jet affected by the pressure difference has flown into the primary vortex zone, which can generate the branch. Before the primary jet starts to separate, only a little primary jet can be ejected from the left vent. After separation, the volume for this ejected jet will increase sharply. However, to be different from the left vent, the phenomena for the right vent is total reverse. This can increase the left thrust and decrease the right thrust.

When the pressure in the primary vortex region tends to be balance, the primary jet flowing into the left output will be reduced gradually. At mean time, the jet flowing into the right output is increasing. The right thrust is also increasing. During the increasing process, the stripping vortex area will be expanded gradually and the primary jet will be hold up. As a result, the collision angle compared with the right output concave wall will be decreased, which can make the strength of shock wave decease. The loss of the energy flowing through the right output channel will also be reduced. In addition, except for the primary jet, it also includes the volume of the right controlling flow. Therefore, the so called as overshoot thrust will be generated in the right output, which will be larger than the steady thrust before switching. 
As shown in Fig.14(b), when the overshoot thrust reached the corresponding peak, the corresponding value will jump up and down irregularly. It is mainly due to the shock wave generated in the right output is extremely unsteady, with the time increasing, the parameters of the right outlet flow will strongly dependent on each time step, resulting in the jumping output thrust.

\section{(c) The changes in the stripping vortex zone}

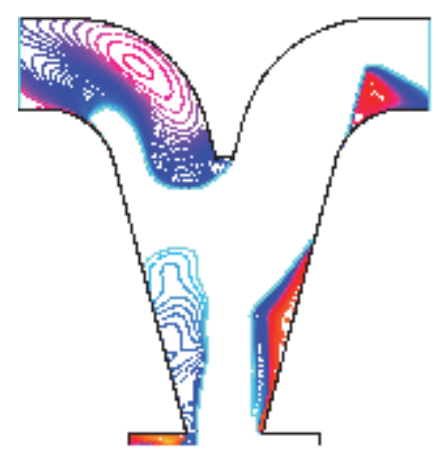

(a) $t=1.6 \times 10^{-4} \mathrm{~s}$

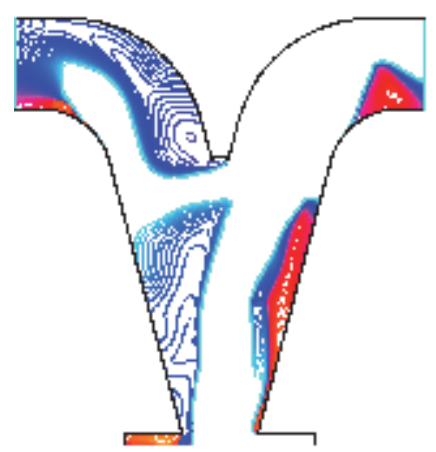

(b) $t=3.2 \times 10^{-4} \mathrm{~s}$

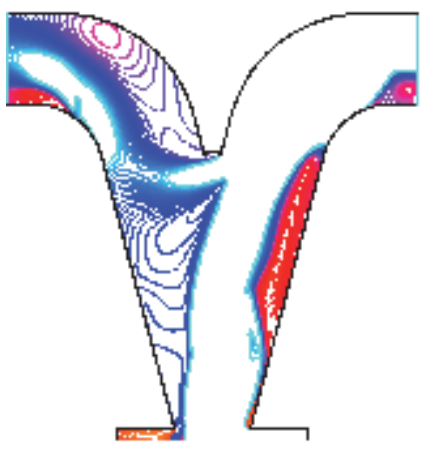

(c) $\mathrm{t}=4.0 \times 10^{-4} \mathrm{~s}$

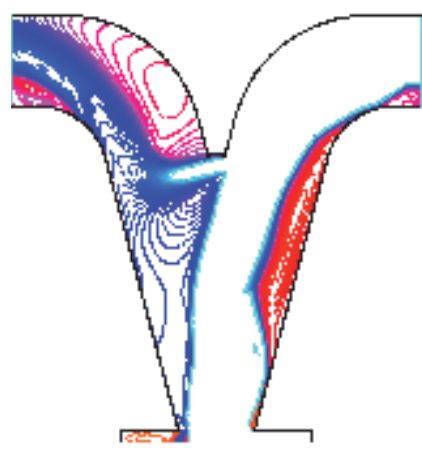

(d) $\mathrm{t}=4.8 \times 10^{-4} \mathrm{~s}$

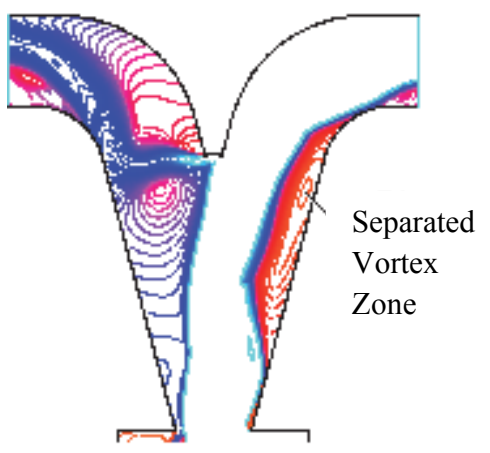

(e) $\mathrm{t}=5.6 \times 10^{-4} \mathrm{~s}$

Fig. 15 The changes in the stripping vortex zone (Stage II)

As shown in Figs.15(a) (e), with the switching time increasing, both the separated and attached points in the stripping vortex zone are moving to the positive $\mathrm{Y}$ direction. And the corresponding zone is also expanding to the longitudinal direction. When $t>3.2$ $\times 10^{-4} \mathrm{~s}$, the position for the separated point has not any change, but the zone will begin to expand to both the longitudinal and transverse directions. When $\mathrm{t}=5.6 \times 10^{-4} \mathrm{~s}$, the stage II will be finished, and the jet has already completely leaved from the right attached wall layer. Compared with the vortex area at $\mathrm{t}=1.6 \times 10^{-4} \mathrm{~s}$, the current area has enlarged about 3 4 times. 


\section{(d) The changes for the Mach number}

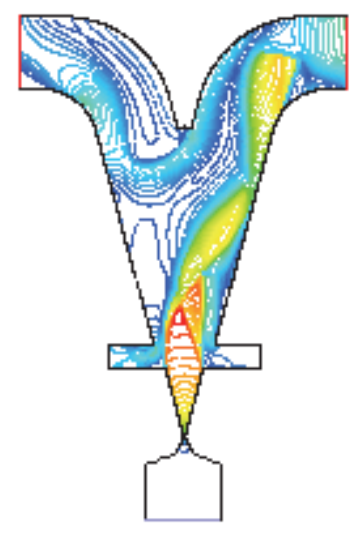

(a) $t=1.6 \times 10^{-4} \mathrm{~s}$

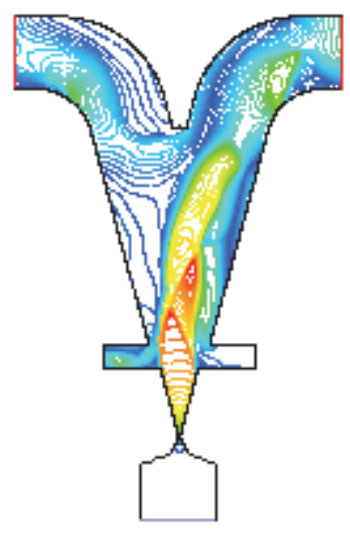

(b) $t=3.2 \times 10^{-4} \mathrm{~s}$

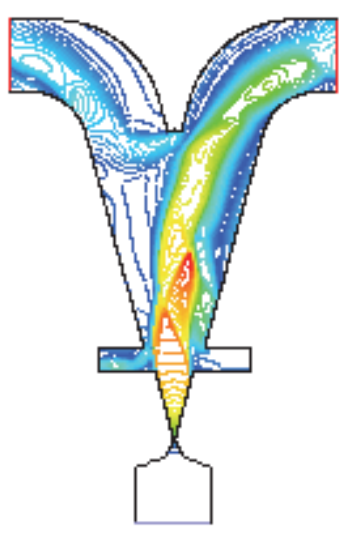

(c) $t=4.8 \times 10^{-4} \mathrm{~s}$

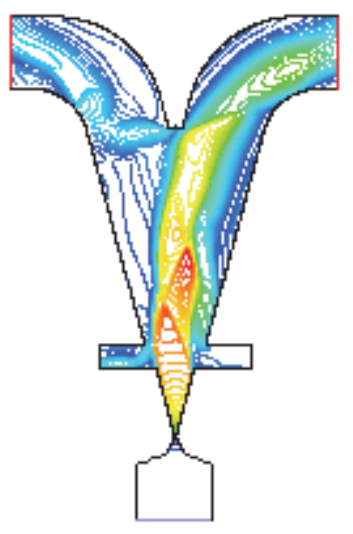

(d) $t=5.6 \times 10^{-4} \mathrm{~s}$

Fig. 16 Contour of the Mach number distribution at different time (Stage II)

As shown in Fig.16, it can be clearly seen the complex flow phenomena, i.e., intersections between shocks, shock reflection on the free shear layer and changes in free shear layers and so on. During the process of the expanding stripping vortex zone and the holding up primary jet, the free shear layer on both sides of the primary jet are continue changing. The left free shear layer not only becomes more smoothly but also moves to the left, and the right shear layer is also expanding and moving to the left.

Both the strength and position for the shock wave within the right output channel are changing with the increasing time. At the very beginning time of the stage II, due to the hysteresis disturbance made by the upstream, both the corresponding strength and position for the shock are showing the same as the phenomena compared with the cases before switching (shown in Fig.16(a)). With the switching time increasing, the strength of the shock has been weakened and the corresponding position has also moved forward to the right output (shown in Figs.16(b) and (c)). When the time reaches the ending moment, the shock in the right output channel has already disappeared. However, due to the presence of the wedge, a new oblique shock has been generated at right output channel (shown in Fig.16(d)). 


\section{(e) The changes of the vortex zone and the transient spatial distribution at different}

\section{time}

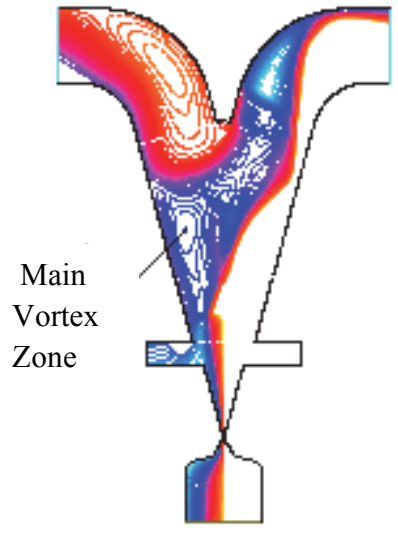

(a) $\mathrm{t}=1.6 \times 10^{-4} \mathrm{~s}$

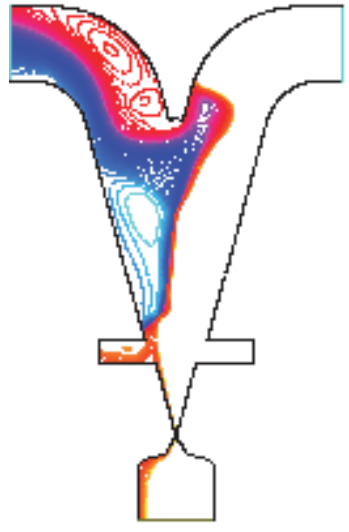

(b) $t=3.2 \times 10^{-4} \mathrm{~s}$

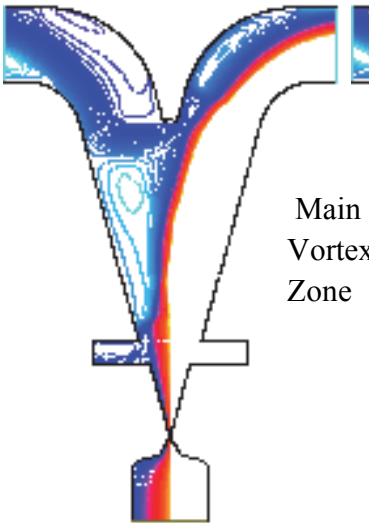

(c) $\mathrm{t}=4.8 \times 10^{-4} \mathrm{~s}$

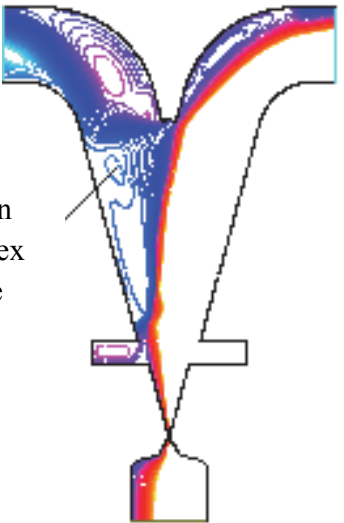

(d) $\mathrm{t}=5.6 \times 10^{-4} \mathrm{~s}$

Fig.17 The changes of the part of the vortex zone (Stage II)

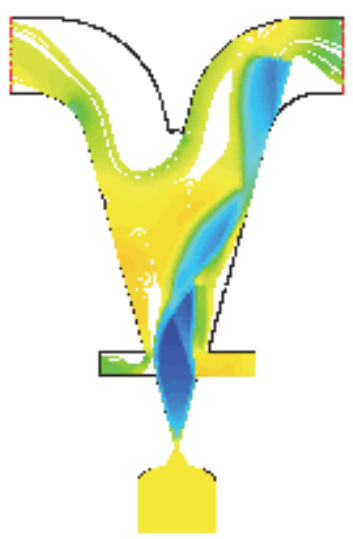

(a) $\mathrm{t}=1.6 \times 10^{-4} \mathrm{~s}$

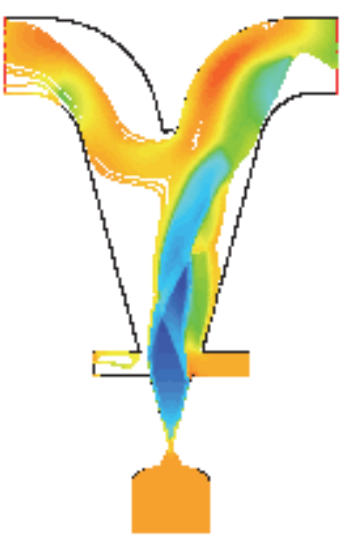

(b) $t=3.2 \times 10^{-4} \mathrm{~s}$

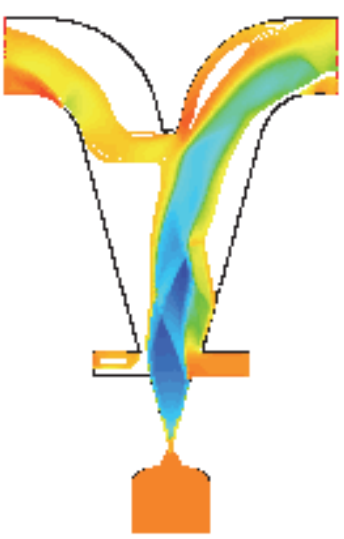

(c) $\mathrm{t}=4.8 \times 10^{-4} \mathrm{~s}$

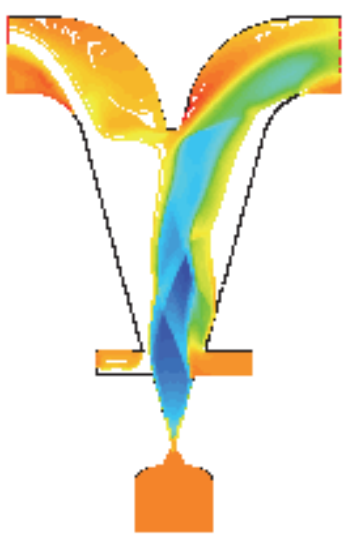

(d) $\mathrm{t}=5.6 \times 10^{-4} \mathrm{~s}$

Fig.18 The transient spatial distribution on the inlet and outlet at different time

As shown in Fig.17, with the time increasing, the primary jet has been hold up and the corresponding area has also been reduced. When $\mathrm{t}>4.8 \times 10^{-4} \mathrm{~s}$, an extremely narrow vortex area has appeared on the right alcove layer. When $\mathrm{t}=1.6 \times 10^{-4} \mathrm{~s}$, there are also some primary jet flow into the primary vortex zone (shown in Fig.17(a)), and small parts of the primary jet have also ejected from the left output. As shown in Fig.17(b), when $\mathrm{t}=3.2 \times 10^{-4} \mathrm{~s}$, the pressure for the primary vortex zone has reached to the balance. There is no primary jet can flow into the zone. When $t=4.8 \times 10^{-4} \mathrm{~s}$, the volume of the primary jet flowing into the zone from the left output has been deceased, and the ejected jet from the right output has been increased. Except this, there also exits some reverse airflow in 
the left and right outputs (shown in Fig.18(c)). When $\mathrm{t}=5.6 \times 10^{-4} \mathrm{~s}$, as shown in Fig.18(d), the area of the reverse airflow in the left output has been expanded, only some extremely small proportions of the primary jet can flow into the left output channel. The corresponding jet will be divided into two main parts, one part will flow into the left concave wall layer, and the other part will be ejected from the left output and then flow into the right output channel. But most of the jet will be ejected from the right output. At the ending time of the stage II, the thrust for the left output has become very small, but the right output can generate an extremely large positive overshoot thrust.

\section{Concluding Remarks}

Based on the unsteady viscous flow simulation, the flow characteristics inside the supersonic jet Element have been investigated numerically. The corresponding switching mechanism and conclusions have been given as follows:

(1) The switching process for the supersonic jet flow is an extremely complex process, which includes the complex shock system evolution, the free shear layers together with the boundary layers evolution and multiple vortex region unsteady evolution etc.

(2) The presence of the switching oblique shock near the control port is not the necessary condition to make the jet deflect, but its formation is good for the early transverse extension of the stripping vortex zone.

(3) The size of the combined thrust is mainly decided by the right output thrust before the main jet flow begins to deflect. After the deflection, the combined thrust will be totally decided by the left output thrust.

(4) A minimum control mass flow rate concept has been proposed and emphasized for a given jet components. Otherwise, the corresponding switching process will not be completed successfully and the direction of the combined thrust will also remain unchanged.

(5) The Viscosity and adverse pressure gradient are the key factors for the occurrence of flow separation to shorten the switching time. 


\section{Acknowledgments}

Financial supports from National Science Foundation of China [Grant number 11602023 and U1430113] are gratefully acknowledged. The authors also would like to thank the reviewers for their helpful suggestions.

\section{References}

[1] M.V. Silnikov, M.V. Chernyshov, and V.N. Uskov, Two-dimensional over-expanded jet flow parameters in supersonic nozzle lip vicinity, Acta Astronautica. 2014(97), $38-41$.

[2] Y.H. Zhao, J.H. Liang, and Y.X. Zhao, Vortex structure and breakup mechanism of gaseous jet in supersonic crossflow with laminar boundary layer, Acta Astronautica. 2016(118), 140-146.

[3] L. Yan, W. Huang, and H. Li, Numerical investigation and optimization on mixing enhancement factors in supersonic jet-to-crossflow flow fields, Acta Astronautica. 2016(127), 321-325.

[4] M.B. Gerdroodbary, M.R. Takami, and H.R. Heidari, Comparison of the single/multi transverse jets under the influence of shock wave in supersonic crossflow, Acta Astronautica. 2016(123), 283-291.

[5] C.M. Hsu, R.F. Huang, and M.E. Loretero, Unsteady flow motions of an oscillating jet in crossflow, Experimental Thermal and Fluid Science. 2014(55), 77-85.

[6] Y.J. Zhang, W.D. Liu, and B. Wang, Investigation of injectant molecular weight effect on the transverse jet characteristics in supersonic crossflow, Acta Astronautica. 2015(115), 423-433.

[7] M.C. Keerthi, and A. Kushari, Effectiveness of vortex generator jets and wall suction on separated flows in serpentine-duct diffuser, Aerospace Science and Technology. 2014(34), 12-19.

[8] K.K. Raja, M.K. Das, and P.R. Kanna, Numerical study of mixed convection in a_two-dimensional_laminar_incompressible_offset jet flow, International Journal of Heat and Mass Transfer. 2009(52), 1023-1035.

[9] R.A. Sawyer, 2-Dimensional Reattaching Jet Flows Including the Effects of Curvature on Entrainment, Journal of Fluid Mechanics. 1963(17), 481-498. 
[10] M.V. Silnikova, M.V. Chernyshov, Incident shock strength evolution in overexpanded jet flow out of rocket nozzle, Acta Astronautica. 2017(online).

[11] G. Sary, G. Dufour, F. Rogier, and K. Kourtzanidis, Modeling and Parametric Study of a Plasma Synthetic Jet for Flow Control, AIAA Journal. 2014(52), 1591-1603.

[12] K.Svensson, P. Rohdin, B. Moshfegh, and M.J. Tummers, Numerical and experimental investigation of the near zone flow field in an array of confluent round jets, International Journal of Heat and Fluid Flow. 2014(46), 127-146.

[13] Y. Xu, G.Q. Zhang, and F.P. Wang, Investigation of the Unsteady Flow for the Supersonic Jet Element, Advanced Materials Research. 2011(201), 2178-2181.

[15] N.N. Smirnov, V.B. Betelin, V.F. Nikitin, L.I. Stamov, D.I. Altoukhov, Accumulation of errors in numerical of chemically reacting gas dynamics. Acta Astronautica, 2015(117), 338-355.

[16] N.N. Smirnov, V.B. Betelin, R.M. Shagaliev, V.F. Nikitin, I.M. Belyakov, Yu.N. Deryuguin, S.V. Aksenov, D.A. Korchazhkin. Hydrogen fuel rocket engines simulation using LOGOS code. International Journal of Hydrogen Energy, 2014(39), 10748-10756. 